\title{
MLSE Receiver for the Dispersive Rayleigh Fading Channel
}

\author{
Wing Seng Leon and Desmond P. Taylor \\ Department of Electrical \& Electronic Engineering \\ University of Canterbury, Christchurch, New Zealand.
}

\begin{abstract}
A maximum likelihood sequence estimator for the dispersive Rayleigh fading channel is developed. Following [1, 2], the MLSE uses a Kalman based channel estimator to acquire the channel parameters necessary to formulate the maximum likelihood metric. However, unlike the MLSE receiver presented in [1, 2], the proposed receiver uses the f-power series channel model $[3,4]$ to formulate the ML metric and the state space representation of the channel and the received samples. For channels with small delay spreads, this approach is advantageous because only a small number of parameters are required to be estimated by the Kalman channel estimator. Simulation results are presented for various channel parameters.
\end{abstract}

\section{Introduction}

Mobile radio channels are usually modeled as time dispersive fading channels due to time varying multipath propagation. The fading causes random phase and amplitude variations in the transmitted signals. Furthermore, due to the dispersive nature of the channel, the receiver observes several delayed and faded versions of the transmitted signals resulting in intersymbol interference (ISI).

The optimum receiver for signals transmitted over such channels is one using maximum likelihood sequence estimation (MLSE). However, channel state information (CSI) must be provided by channel estimators for MLSE. To date, there are several proposed MLSE receivers for time dispersive fading channels [1, 2, 5]. Lodge and Moher [1] briefly described a MLSE receiver for a general fading channel using the Viterbi Algorithm (VA) and a bank of Kalman filters to continuously estimate the CSI. This is further developed by Dai and Shwedyk [2] explicitly for the time dispersive Rayleigh fading channel. Most recently, in [5], an innovations-based MLSE receiver was described with CSI supplied by a set of time invariant linear filters. The implementation of these receivers require the knowledge of channel statistics.

In these previous studies, the channel has been modeled as a tapped delay line (TDL) with randomly time varying tap gains. However, the channel model need not necessarily be restricted to a TDL configuration. The channel model described in $[3,4,6]$, results from the Taylor's series expansion of the channel transfer function. This is known as the f-power series model in [3] or the reduced dimensionality model in [4]. It has been shown that a truncated f-power series with only the first few terms is necessary to define a channel with small delay spread quite accurately [4].

In this paper, we present an MLSE receiver for a time dispersive Rayleigh fading channel following the approach of $[1,2]$ using Kalman filters to estimate the CSI. Here, the channel model is used in the design of the receiver is the f-power series model. It is optimum if the order of the channel order is sufficient. The receiver is implemented using the VA and per survivor processing (PSP) [7] to reduce complexity.
In other receivers, the entire composite channel impulse response (CIR) which may span many symbols is estimated. The number of estimated parameters is further increased for multi-sampling receivers. The proposed receiver only estimates the parameters of the fading channel. Since a small number of channel parameters are used in a truncated fpower series for a channel with a relatively small delay spread, the resulting Kalman channel estimator is also simplified.

This paper is organized as follows. Section 2 shows describes the signal and channel models. In section 3, the MLSE receiver is described. The state and measurement models of the sampled received signal are discussed in section 4. The results of computer simulations of communication systems using the proposed receiver are presented in section 5. Finally, some concluding remarks are provided in section 6 .

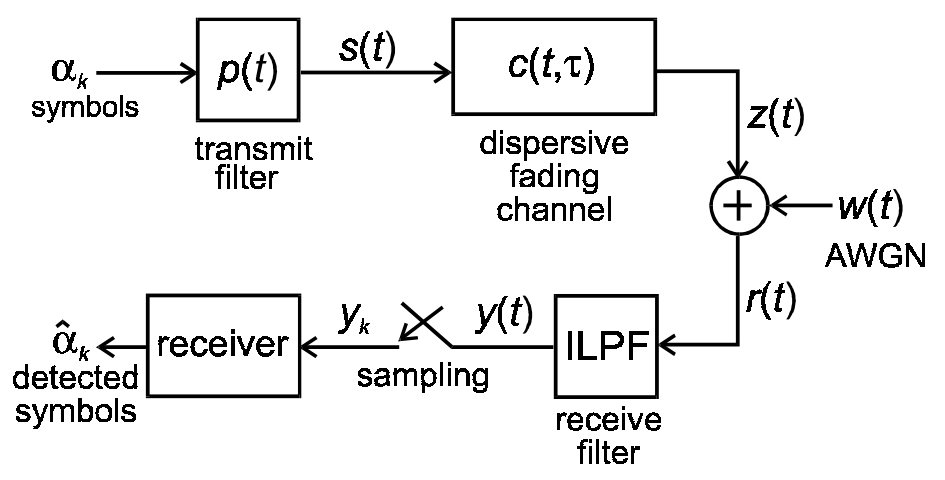

Figure 1. Block diagram of the communication system.

\section{Channel and Signal Models}

Figure 1 shows the complex baseband model of a communication system with a dispersive Rayleigh fading channel. The transmitter consists of a symbol source which outputs data symbols of a linear modulation scheme. The transmitted complex baseband signal is

$$
s(t)=\sum_{l=1}^{N} \alpha_{l} p(t-l T)
$$

where $p(t)$ is the impulse response of the transmit filter with normalized energy of unity, $1 / T$ is the symbol rate and $\left\{\alpha_{l}\right\}$ are uncorrelated data symbols.

Following the f-power series derivation in $[3,6]$ and setting the mean delay to zero, the signal component of the received signal is written as

$$
z(t)=\sum_{n=0}^{m} T_{n}(t) \frac{d^{n}[s(t)]}{d t^{n}}
$$

where $T_{n}(t)$ is the time selective coefficient of the $n$-th term of the series. The model is shown in figure 2. This signal model is derived from the Taylor's series expansion of the time varying channel transfer function $[3,6]$. Although the exact representation of the channel requires a series with an infinite number of terms, we have truncated the series in (2) and limited it to the first $m+1$ terms. For a channel with small delay 
spread, only the first few terms of the f-power series are necessary to model the channel to a high degree of accuracy [4]. From (2), it can be seen that for any given signal $s(t)$, the channel is completely defined by the time selective coefficients.

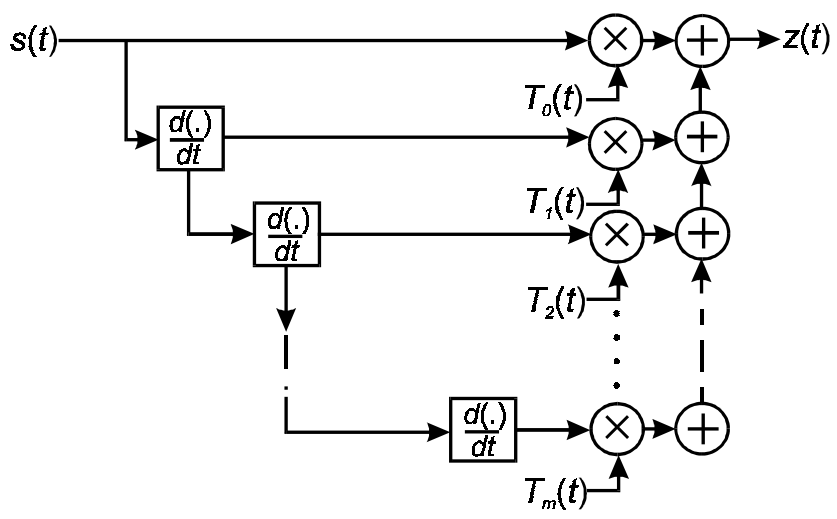

Figure 2. The $m$-th order $\mathrm{f}$-power series model of a dispersive fading channel.

To understand the nature of the time selective coefficients, consider the a typical multipath fading channel. The discrete-time CIR using a conventional TDL model is

$$
c(t, \tau)=\sum_{i=1}^{J} \varphi_{i}(t) \delta\left(\tau-\tau_{i}\right)
$$

where $\varphi_{i}(t)$ is the zero mean complex Gaussian fading process of the $i$-th propagation path or ray. As in [4], we assume a wide sense stationary uncorrelated scattering model for the fading channel and isotropic scattering in each path. Therefore, the autocorrelation of the coefficients $\varphi_{i}(t)$ is

$$
\begin{gathered}
\frac{1}{2} E\left[\varphi_{i}(t) \varphi_{j}^{*}(t+\xi)\right]=\left\{\begin{array}{cc}
\phi_{i}(\xi) & \text { for } i=j \\
0 & \text { otherwise }
\end{array}\right. \\
\phi_{i}(\xi)=\sigma_{i}^{2} \rho(\xi) \\
\rho(\xi)=J_{0}\left(2 \pi f_{D} \xi\right)
\end{gathered}
$$

where superscript $*$ denotes complex conjugate, $\sigma_{i}^{2}$ is the average power contributed by the $i$-th delay path, $f_{D}$ is the maximum Doppler frequency and $J_{0}(\bullet)$ is the zeroth order Bessel function.

The $n$-th time selective coefficient $T_{n}(t)$ is related to the CIR (3) by

$$
T_{n}(t)=\sum_{i=1}^{J} \frac{\left(-\tau_{i}\right)^{n}}{n !} \varphi_{i}(t)
$$

Since the $\varphi_{i}(t)$ 's are zero mean complex Gaussian r.v., it follows that the time selective coefficients $T_{n}(t)$ 's are also zero mean complex Gaussian r.v. Using (4) to (7), the autocorrelation functions of the time selective coefficients can also be defined.

The channel output $z(t)$ is corrupted by zero mean additive white Gaussian noise $w(t)$ with power density of $N_{o}$. Therefore, the received signal is

$$
r(t)=z(t)+w(t)
$$

The receiver filter (ideal low pass filter) with bandwidth $B_{w}$, must accommodate the entire bandwidth of $z(t)$ so that the receiver can obtain sufficient statistics to decode the transmitted signal. Following [2], the filtered received samples are given by

$$
y\left(k T_{s}\right)=\sum_{n=0}^{m} T_{n}\left(k T_{s}\right) \sum_{l=1}^{N} \alpha_{l} p^{(n)}\left(k T_{s}-l T\right)+n\left(k T_{s}\right)
$$

where $T_{s}=1 / f_{s}$ is the sampling period, $f_{s}=2 B_{w}$ and the superscript $(n)$ denotes the $n$-th derivative. The filtered noise samples $n\left(k T_{s}\right)$ are uncorrelated Gaussian random variables with variance $N_{o}^{w}=f_{s} N_{o}$. It is convenient to let $T=\gamma T_{s}$ where $\gamma$ is an integer which represents the number of samples per symbol duration. For brevity, the reference to the sampling period $T_{s}$ is dropped and (9) becomes

$$
y_{k}=\sum_{n=0}^{m} T_{n}(k) s_{k}^{(n)}+n_{k}
$$

where

$$
s_{k}^{(n)}=\sum_{l=1}^{N} \alpha_{l} p^{(n)}\left(k T_{s}-l T\right)
$$

\section{The Maximum Likelihood Sequence Estimator}

Assuming a $q$-ary modulation format and a sequence length of $N$, there are $g=q^{N}$ possible distinct sequences. Let $\underline{\alpha}(\zeta)$ denote an $N$-dimension complex vector where $\alpha_{l}(\zeta)$ its $l$-th element and the term which indexes each of the sequence is $\zeta$ $=1,2, \ldots, g$. Therefore the sequences are represented by $\underline{\alpha}(1), \underline{\alpha}(2), \cdots, \underline{\alpha}(g)$. We define a $(\gamma N \times m+1)$ signal matrix $\mathbf{S}(\zeta)$ and its $k$-th row is

$$
\mathbf{s}_{\mathbf{k}}(\zeta)=\left[s_{k}^{(0)}(\zeta), s_{k}^{(1)}(\zeta), \cdots, s_{k}^{(m)}(\zeta)\right]
$$

From (11) and (12), we see that for every hypothesized symbol sequence $\underline{\alpha}(\zeta)$, there is a corresponding signal matrix $\mathbf{S}(\zeta)$. Let the received signal vector be $\mathbf{y} \in \mathrm{C}^{\gamma N}$ and its $k$-th element be $y_{k}$, the maximum likelihood (ML) decision is $\mathbf{S}(\chi)$ and correspondingly $\underline{\alpha}(\chi)$, if

$$
p[\mathbf{y} \mid \mathbf{S}(\chi)]>p[\mathbf{y} \mid \mathbf{S}(\zeta)], \zeta=1,2, \cdots, g ; \chi \neq \zeta(13)
$$

Since the received samples $y_{k}$ have complex Gaussian distributions, the ML metric is $[1,2]$

$$
\begin{gathered}
\Lambda_{N}^{\zeta}(\mathbf{y})=\sum_{k=1}^{\gamma N}\left[\frac{\left|y_{k}-y_{k \mid k-1}(\zeta)\right|^{2}}{\tilde{\sigma}_{k \mid k-1}^{2}(\zeta)}+\log \left(\tilde{\sigma}_{k \mid k-1}^{2}(\zeta)\right)\right] \\
\zeta=1,2, \cdots, g ; \chi \neq \zeta
\end{gathered}
$$


where $y_{k \mid k-1}(\zeta)$ and $\tilde{\sigma}_{k \mid k-1}^{2}(\zeta)$ are the mean and variance conditioned on the previous $k-1$ samples respectively for a given hypothesis $\zeta$. The ML receiver computes the metric in (14) for all possible hypotheses and selects the data sequence which corresponds to the hypothesis yielding the minimum metric. Using the approach in [2], the MLSE receiver is easily implemented using the Viterbi algorithm.

\section{Kalman Channel Estimator and State Space Representation}

It is seen in (14) that the metric computation requires knowledge of the conditional mean $y_{k \mid k-1}(\zeta)$ and variance $\tilde{\sigma}_{k \mid k-1}^{2}(\zeta)$ (channel state information). As in [1, 2], a Kalman filter is used as the channel state estimator to compute the conditional mean and variance for each hypothesis. To implement the Kalman filter, a state space model of the channel and a measurement model of the received samples must be defined $[8,9]$. The state model has the general form

$$
\mathbf{x}_{\mathbf{k}+1}=\mathbf{A} \mathbf{x}_{\mathbf{k}}+\mathbf{B} \mathbf{v}_{\mathbf{k}}
$$

where $\mathbf{x}_{\mathbf{k}}$ is the state process, $\mathbf{A}$ is the state transition matrix, $\mathbf{B}$ is the correlation matrix of the state processes and $\mathbf{v}_{\mathbf{k}}$ is the process noise vector. The matrices $\mathbf{A}$ and $\mathbf{B}$ are time invariant. The autocorrelation matrix of the process noise vector $\mathbf{v}_{\mathbf{k}}$ is an identity matrix of the appropriate size. For a Rayleigh fading channel, $\mathbf{v}_{\mathbf{k}}$ is a complex Gaussian vector. Using (10), the measurement or observation model is written as

$$
y_{k}=\mathbf{s}_{\mathbf{k}} \mathbf{x}_{\mathbf{k}}+n_{k}
$$

where $\mathbf{x}_{\mathbf{k}}=\left[T_{\mathrm{o}}(k), T_{1}(k), \ldots, T_{m}(k)\right]^{\mathrm{T}}$ and the superscript $\mathrm{T}$ denotes transposition. For a given hypothesis $\gamma, \mathbf{s}_{\mathbf{k}}=\mathbf{s}_{\mathbf{k}}(\gamma)$.

In order to implement the filter, $\mathbf{A}$ and $\mathbf{B}$ must be known. To formulate these matrices, we assume that the fading process associated with each delay path is an autoregressive (AR) process of order $M$. If the process is not AR, then it may be approximated using an AR process of a suitable order [1]. From (4) and (5), we define

$$
\varphi_{i}(k)=\sigma_{i} \hat{\varphi}_{i}(k)
$$

where $\hat{\varphi}_{i}(k)$ is the normalized complex gain of the $i$-th path and has unity average power. Therefore, the fading process may be written as

$$
\sigma_{i} \hat{\varphi}_{i}(k)=\sum_{r=1}^{M} a_{r} \sigma_{i} \hat{\varphi}_{i}(k-r)+\sigma_{i} v_{i}(k)
$$

where $a_{r}$ is the $r$-th tap weight of the filter which produces the AR process associated with the $i$-th delay path. The complex Gaussian noise $v_{i}(k)$ driving the AR process has the property that

$$
E\left[v_{i}(k) v_{j} *(l)\right]=\left\{\begin{array}{cc}
1 & \text { for } k=l \text { and } i=j \\
0 & \text { otherwise }
\end{array}\right.
$$

From (7), the time selective coefficient $T_{n}(k)$ is written as

$$
T_{n}(k)=\sum_{i=1}^{J} \frac{\left(-\tau_{i}\right)^{n}}{n !} \sigma_{i} \hat{\varphi}_{i}(k)
$$

Substituting (18) into (20), the time selective coefficient becomes

$$
T_{n}(k)=\sum_{i=1}^{J} \frac{\left(-\tau_{i}\right)^{n}}{n !}\left\{\sum_{r=1}^{M} \sigma_{i} a_{r} \hat{\varphi}_{i}(k-r)+\sigma_{i} v_{i}(k)\right\}
$$

Interchanging the order of summation and expanding, (21) becomes

$$
\begin{aligned}
T_{n}(k)= & \sum_{r=1}^{M} a_{r}\left\{\sum_{i=1}^{J} \frac{\left(-\tau_{i}\right)^{n}}{n !} \sigma_{i} \hat{\varphi}_{i}(k-r)\right\} \\
& +\sum_{i=1}^{J} \frac{\left(-\tau_{i}\right)^{n}}{n !} \sigma_{i} v_{i}(k)
\end{aligned}
$$

The term within the \{\} parenthesis, by the definition in (22), is the time selective coefficient at time index $(k-r)$. Therefore, (22) simplifies to

$$
T_{n}(k)=\sum_{r=1}^{M} a_{r} T_{n}(k-r)+\sum_{i=1}^{J} b_{n, i} v_{i}(k)
$$

where

$$
b_{n, i}=\frac{\left(-\tau_{i}\right)^{n}}{n !} \sigma_{i}
$$

It is seen in (23) that the time selective coefficient $T_{n}(k)$ is also an AR process driven by uncorrelated Gaussian noise. The autocorrelation function of this process is the same as that of the original process since the tap weights $a_{r}$ remain unaffected. However, the process noise is now made up of a linear sum of complex white noise from $J$ uncorrelated noise sources, each weighted by $b_{n, i}$.

To establish the matrices $\mathbf{A}$ and $\mathbf{B}$, we augment the state vector $\mathbf{x}_{\mathbf{k}}$ in (16) as

$$
\begin{aligned}
& \mathbf{x}_{\mathbf{k}}=\left[T_{0}(k), \cdots, T_{0}(k-M+1), T_{1}(k), \cdots,\right. \\
& \left.T_{1}(k-M+1), \cdots, T_{m}(k), \cdots, T_{m}(k-M+1)\right]^{\mathrm{T}}{ }^{(25}
\end{aligned}
$$

which is a $(m+1) M \times 1$ vector. It is known that the state transition matrix for the $M$-th order AR process associated with the $n$-th order time selective coefficient of the f-power series may be written as

$$
\Phi_{n}=\left[\begin{array}{cccc}
a_{1} & a_{2} & \cdots & a_{M} \\
1 & 0 & \cdots & 0 \\
0 & \ddots & \ddots & 0 \\
0 & 0 & 1 & 0
\end{array}\right]
$$

Therefore, the state transition matrix of the f-power series model is written as 


$$
\mathbf{A}=\left[\begin{array}{cccc}
\Phi_{0} & 0 & \cdots & 0 \\
0 & \Phi_{1} & \ddots & \vdots \\
\vdots & \ddots & \ddots & 0 \\
0 & \cdots & 0 & \Phi_{m}
\end{array}\right]
$$

For an $m$-th order f-power series with $M$-th order channel fading AR processes, the state transition matrix $\mathbf{A}$ is $(m+1) M \mathrm{x}$ $(m+1) M$. The $\mathbf{B}$ matrix is defined as

$$
\mathbf{B}=\left[\begin{array}{cccc}
b_{0,1} & b_{0,2} & \cdots & b_{0, J} \\
0 & 0 & \cdots & 0 \\
\vdots & \vdots & \ddots & \vdots \\
b_{1,1} & b_{1,2} & \cdots & b_{1, J} \\
0 & 0 & \cdots & 0 \\
\vdots & \vdots & \ddots & \vdots \\
b_{m, 1} & b_{m, 2} & \cdots & b_{m, J} \\
\vdots & \vdots & \cdots & \vdots
\end{array}\right]
$$

This is a $(m+1) \times J$ matrix with $(M-1)$ rows of zeros inserted between the rows of $b_{n, i}$. The process noise vector $\mathbf{v}_{\mathbf{k}}$ is therefore defined as a $J \times 1$ column vector. Finally, to complete the model, the measurement vector is augmented to be dimensionally consistent with the augmented state vector in (25). For a given transmitted data sequence, the augmented measurement vector is

$$
\mathbf{s}_{\mathbf{k}}=\left[s_{k}^{(0)}, 0 \quad \cdots \quad 0, s_{k}^{(1)}, 0 \cdots \cdots, s_{k}^{(m)}, 0 \cdots\right]
$$

where $(M-1)$ zeros are inserted between the elements of the original vector in (12) to yield a $1 \times(m+1) M$ row vector.

With the above state space model of the channel and received samples, the conditional mean and variance of the ML metric is estimated using the Kalman filtering algorithm as $[8,9]$

$$
\begin{aligned}
\hat{\mathbf{x}}_{\mathbf{k}+\mathbf{1}} & =\left(\mathbf{A}-\mathbf{G}_{\mathbf{k}} \mathbf{s}_{\mathbf{k}}\right) \hat{\mathbf{x}}_{\mathbf{k}}+\mathbf{G}_{\mathbf{k}} y_{k} \\
\mathbf{G}_{\mathbf{k}}= & \mathbf{A} \mathbf{K}_{\mathbf{k}} \mathbf{s}_{\mathbf{k}}^{H}\left(\mathbf{s}_{\mathbf{k}} \mathbf{K}_{\mathbf{k}} \mathbf{s}_{\mathbf{k}}^{H}+N_{o}^{w}\right)^{-1} \\
\mathbf{K}_{\mathbf{k}+\mathbf{1}} & =\left(\mathbf{A}-\mathbf{G}_{\mathbf{k}} \mathbf{s}_{\mathbf{k}}\right) \mathbf{K}_{\mathbf{k}} \mathbf{A}^{H}+\mathbf{B B} \mathbf{B}^{H} \\
& y_{k \mid k-1}(\zeta)=\mathbf{s}_{\mathbf{k}} \hat{\mathbf{x}}_{\mathbf{k}} \\
& \tilde{\boldsymbol{\sigma}}_{k \mid k-1}^{2}(\zeta)=\mathbf{s}_{\mathbf{k}} \mathbf{K}_{\mathbf{k}} \mathbf{s}_{\mathbf{k}}^{H}+N_{o}^{w}
\end{aligned}
$$

where $\hat{\mathbf{x}}_{\mathbf{k}}$ is the estimated state vector, $\mathbf{G}_{\mathbf{k}}$ the Kalman gain vector and $\mathbf{K}_{\mathbf{k}}$ the error covariance matrix.

The state space representation described above is mathematically appealing. The cross-correlations among the time selective coefficients $T_{n}(k)$ with $n=0,1,2, \ldots, m$ is contained within the matrix $\mathbf{B}$ and the time autocorrelation of each of the time selective coefficients is implicitly described by the state transition matrix. This representation can be applied to a general f-power series model of order $m$ and a channel with $J$ rays. Therefore, if the series is truncated to a reasonable order, e.g. $m=2, J=2$ or 3 , and if an AR description of the channel fading process is not of high order, then the matrices and vectors in model will be of manageable size.

For channels with small delay spreads, a truncated fpower series with a few terms is adequate in modeling the channel. By formulating the state space representation as shown, only a small number of parameters are internally processed by the Kalman filter. This is an improvement over other receivers using a TDL channel model $[1,2]$ because the entire composite CIR is estimated.

\section{Simulation Results}

Monte Carlo simulation was performed to evaluate the performance of the Kalman based MLSE receiver described above. The receiver was implemented using the Viterbi algorithm and per survivor processing to reduce complexity.

Uncoded 4-PSK modulation format was adopted for the simulation and the bit energy is $E_{b}$. The impulse response of the transmit filter is a full raised cosine with 50\% roll-off [10]. A three ray $(J=3)$ multipath channel with a uniform delay power profile was used. The fading associated with each path is generated as in [11]. It is assumed that the receiver has complete knowledge of the second order statistics of the channel. The receiver assumes that the channel fading process is a 4-th order $(M=4)$ AR process and employs a quadratic $(m=$ 2) f-power series model. The oversampling rate is $\gamma=2$ samples per symbol.

The bit error rates (BER) obtained by simulation are presented in figure 3 and 4 . Two fade rates, $f_{D} T=0.1,0.02$ and three normalized maximum delay spreads, $\tau_{\max } / T=0.16$, $0.20,0.25$ were used. It is seen that the Kalman based MLSE receiver performs slightly better in the faster fading environment. This is attributed to the increased implicit time diversity of the faster fading channel. It is also obvious that the performance of the receiver degrades for a channel with a larger delay spread. Although an increased channel delay spread would imply an equivalent increase in implicit frequency diversity, the effects may not be significant. As shown in [4], the modeling error of the f-power series model and the delay spread of the channel are related. For a given order of the $f-$ power series model, the modeling error increases with increasing channel delay spread. Therefore, the worsening of the BER results as channel delay spread is increased may be attributed to the increased modeling error. However, there are no observable error floors for the range of fade rates, delay spreads and SNR simulated. An important point to note that the receiver assuming a quadratic f-power series is able to cope with channels with maximum delay spread up to $25 \%$ of the symbol duration.

\section{Conclusion}

An MLSE receiver with a Kalman filtering channel estimator has been proposed in this paper. Unlike existing receivers, this receiver assumes that the channel is modeled as an $\mathrm{f}$ power series. It is optimum if the order of the model is sufficient to accurately represent the channel. A channel with a small delay spread will only require a f-power series model of a small order. Therefore, only a small number of parameters need to be estimated by the Kalman channel estimator. This is an advantage over conventional receivers assuming a TDL channel model which usually estimates the entire CIR. Using 
an f-power series channel model, a state space representation of the channel and received samples is formulated so that the Kalman filtering algorithm can be used to obtain the necessary channel state information for the MLSE. To reduce complexity, the receiver is implemented using the Viterbi algorithm and per survivor processing.

Simulated BER performance of the receiver is presented. The results are encouraging because no irreducible error floors are apparent in the BER curves. Furthermore, the receiver, assuming a quadratic f-power series channel model, is able to cope with channels with maximum delay spreads of at least $25 \%$ of symbol duration. The BER performances of the faster fading channels are better due to the implicit time diversity of the channel. However, the performance of the receiver is degraded for channels with larger delay spreads. This may be due to the increased significance of the modeling error.

\section{References}

[1] J.H. Lodge and M.L. Moher, "Maximum Likelihood Sequence Estimation of CPM Signals Transmitted over Rayleigh Flat-Fading Channels", IEEE Trans. Commun., vol. 38, pp. 787-794, June 1990.

[2] Q. Dai and E. Shwedyk, "Detection of Bandlimited Signals over Frequency Selective Rayleigh Fading Channels", IEEE Trans. Commun., vol. 42, pp. 941950, February/March/April 1994.

[3] P.A. Bello, "Characterization of Randomly TimeVariant Linear Channels", IEEE Trans. Comm. Sys., vol. 11, pp. 360-393, December 1963.

[4] G. Deng, J. Cavers and P. Ho, "A Reduced Dimensionality Propagation Model for Frequency Selective Rayleigh Fading Channels", ICC, Seattle, 1995, pp. 1158-1162.

[5] X. Yu and S. Pasupathy, "Innovation-based MLSE for Rayleigh Fading Channels", IEEE Trans. Commun. vol. 43, pp. 1534-1544, February/March/April 1995.

[6] W.S. Leon, “The Reception of QPSK Signals Transmitted Over Dispersive Fading Channels Using the fPower Series Channel Model", M.E. Thesis, University of Canterbury, Christchurch, New Zealand, 1995.

[7] R. Raheli, A. Polydoros, C-K. Tzou, "The Principle of Per-Survivor Processing: A General Approach to Approximate and Adaptive MLSE”, GLOBECOM, Phoenix, 1991, pp. 1170-1175.

[8] S. Haykin, Adaptive Filter Theory, 2nd ed., Prentice Hall, Englewood Cliffs, New Jersey, 1991.

[9] B.D.O. Anderson and J.B. Moore, Optimal Filtering, Prentice-Hall, Englewood Cliffs, New Jersey, 1979.

[10] S. Haykin, Communication Systems, 2nd ed., John Wiley and Sons, New York, 1983.

[11] D. Verdin and T.C. Tozer, "Generating a Fading Process for the Simulation of Land-Mobile Radio Communications", Electronics Letters, vol. 29, no. 23, pp. 2011-2012, 11 November 1993.

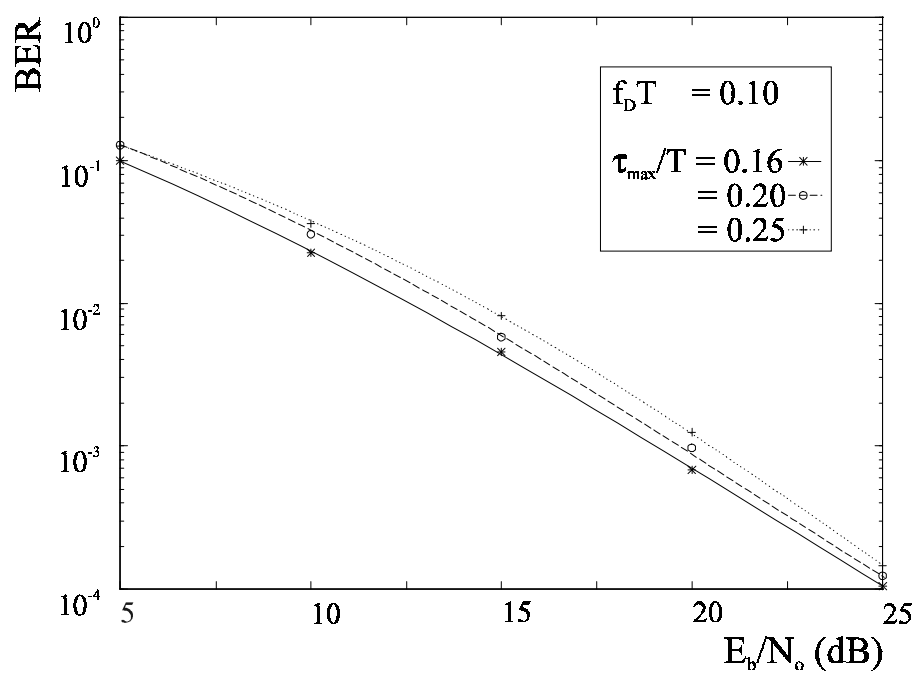

Figure 3. BER curves of the proposed Kalman based MLSE receiver for uncoded 4-PSK. $f_{D} T=0.1$ and $\tau_{\max } / T=0.16$, $0.20,0.25$.

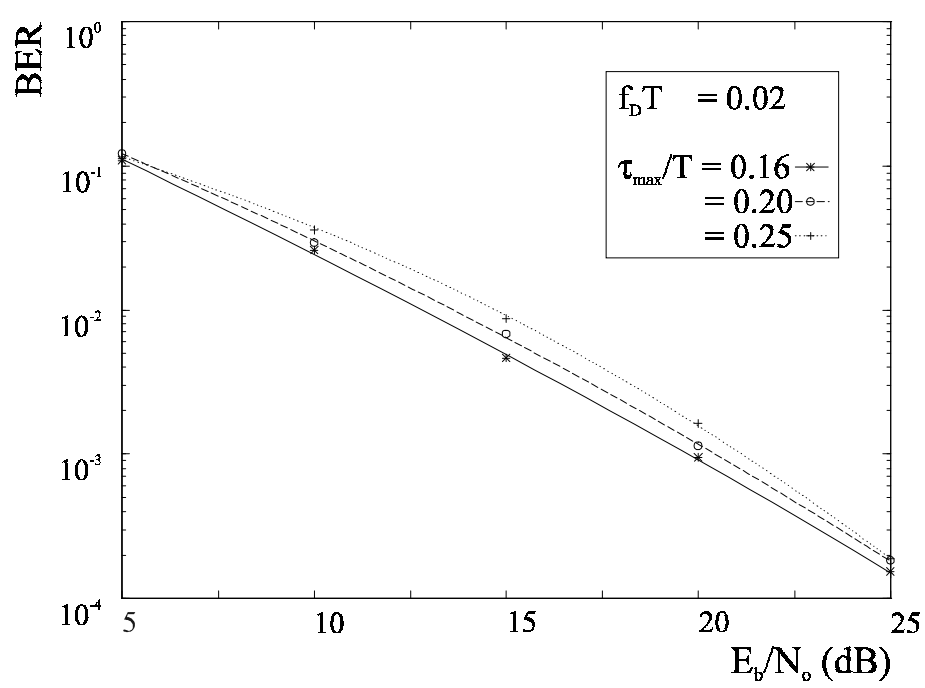

Figure 4. BER curves of the proposed Kalman based MLSE receiver for uncoded 4-PSK. $f_{D} T=0.02$ and $\tau_{\max } / T=0.16$, $0.20,0.25$. 